\title{
Emergent Properties of Proteostasis in Managing Cystic Fibrosis
}

\author{
William E. Balch ${ }^{1,2,3,4}$, Daniela M. Roth ${ }^{1}$, and Darren M. Hutt ${ }^{1}$ \\ ${ }^{1}$ Department of Cell Biology, The Scripps Research Institute, La Jolla, California 92037 \\ ${ }^{2}$ The Skaggs Institute for Chemical Biology, The Scripps Research Institute, La Jolla, California 92037 \\ ${ }^{3}$ Department of Chemical Physiology, The Scripps Research Institute, La Jolla, California 92037 \\ ${ }^{4}$ The Institute for Childhood and Neglected Diseases, The Scripps Research Institute, La Jolla, California 92037 \\ Correspondence: webalch@scripps.edu
}

Cystic fibrosis (CF) is a consequence of defective recognition of the multimembrane spanning protein cystic fibrosis conductance transmembrane regulator (CFTR) by the protein homeostasis or proteostasis network (PN) (Hutt and Balch (2010). Like many variant proteins triggering misfolding diseases, mutant CFTR has a complex folding and membrane trafficking itinerary that is managed by the $\mathrm{PN}$ to maintain proteome balance and this balance is disrupted in human disease. The biological pathways dictating the folding and function of CFTR in health and disease are being studied by numerous investigators, providing a unique opportunity to begin to understand and therapeutically address the role of the PN in disease onset, and its progression during aging. We discuss the general concept that therapeutic management of the emergent properties of the PN to control the energetics of CFTR folding biology may provide significant clinical benefit.

\section{CYSTIC FIBROSIS AS A SYSTEMS DISEASE}

Cystic fibrosis (CF) is an inherited disorder caused by mutations affecting the function of the cystic fibrosis transmembrane conductance regulator (CFTR), a cAMP gated chloride/bicarbonate channel that regulates the hydration of the epithelial surfaces found in multiple tissues including the liver, pancreas, intestine, and lung (Riordan 2008). $\mathrm{CF}$ can be viewed as a triad of pathologies (Fig. 1), which, at its root, is a loss-of-function disease in response to uncoupling of the CFTR peptide chain stability from folding pathways defined by the protein homeostasis or proteostasis network (PN) of the cell (Balch et al. 2008; Hutt and Balch 2010) (Fig. 2). The pathophysiological CF triad not only includes a folding defect leading to loss of salt balance, but loss of surface hydration and reduced mucociliary clearance, which trigger a chronic inflammatory response, leading to a multiple organ failure phenotype. It is the triad of these pathological events that is driven by integrated biological networks that are sensitive to the status of the CFTR fold that we hope to regulate through therapeutic management of the PN to, in essence, use normal biology to correct disease biology.

Editors: Richard Morimoto, Jeffrey Kelly, and Dennis Selkoe

Additional Perspectives on Protein Homeostasis available at www.cshperspectives.org

Copyright (C) 2011 Cold Spring Harbor Laboratory Press; all rights reserved; doi: 10.1101/cshperspect.a004499

Cite this article as Cold Spring Harb Perspect Biol 2011;3:a004499 
W.E. Balch et al.

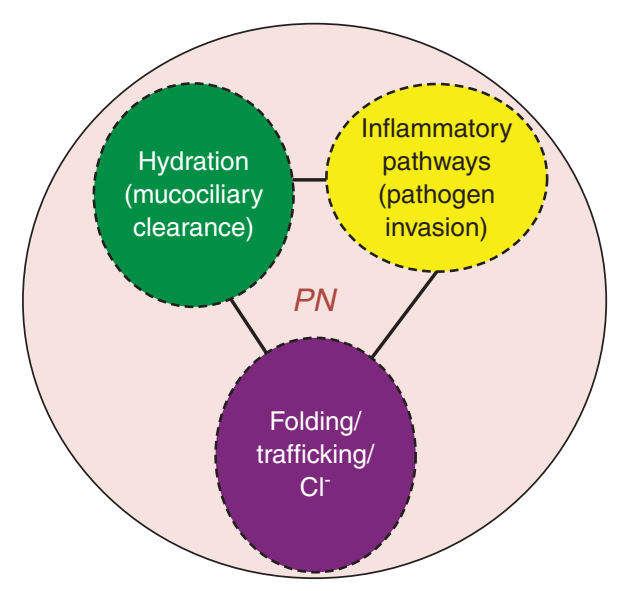

Figure 1. The pathological Cystic Fibrosis triad. Illustrated are the three principle pathologic features observed in the clinic that define the CF disease triad in response to vCFTR. Each feature is likely to be subject to PN management either indirectly through the CFTR fold, or directly by PN components (Fig. 2).

To date $>1600$ disease-causing mutations have been identified in the CFTR gene (referred to as variant CFTR [vCFTR]). These fall into six classes (I-VI) that represent a breakdown in CFTR folding and function at different stations in its trafficking itinerary through the exocytic and endocytic pathways, and in channel function (Becq 2010) (Fig. 3A). As might be expected, presentation of these different mutations in the clinic is quite diverse, ranging from mild to severe disease pathology, reflecting the location of the mutation in the vCFTR peptide chain sequence, its impact on folding energetics at different steps of its trafficking itinerary, and its indirect or direct effect on channel activity and protein interactions seen in the CFTR network (Wang et al. 2006; Wright et al. 2009). Interestingly, the loss of Phe 508 ( $\Delta$ F508 vCFTR), a mutation affecting $>90 \%$ of CF patients, presents and progresses in the clinic with a wide range of pathologies and time frames. This emphasizes the importance of a multiplicity of modifiers (Collaco and Cutting 2008), likely to include as yet many unidentified PN components, that set the stage not only for the initial disease etiology observed at birth, but numerous aging-related changes associated with loss of vCFTR function leading to the inevitable progressive loss of tissue function (Fig. 1).

The questions we need to address to better understand the CFTR folding/misfolding problem and for generating effective therapeutics to ameliorate disease through modulation of the PN are: (1) Are wild-type (WT) CFTR and vCFTR affected similarly by the composition and activity of the local cellular PN? (2) Do we have suitable models to characterize the role of the PN in disease? (3) What is the maximal level of rescue that we can hope to achieve by manipulating the PN? and finally, (4) Is targeting the CFTR PN sufficient or do we need to develop additional strategies that focus on non-CFTR targets to maximize patient benefit with respect to the CF disease triad (Fig. 1). In other words, can we use the dynamic and emergent properties of the PN to efficiently manifest correction of multiple disease etiologies associated with $\mathrm{CF}$ ?

\section{THE PN AS A FRAMEWORK FOR CF DISEASE MANAGEMENT}

At least a thousand components contribute to the PN (Fig. 2) (Balch et al. 2008; Powers et al. 2009; Hutt and Balch 2010). These include the Hsp/Hsc70 and Hsp90 chaperone systems whose normal activities are regulated by numerous signaling pathways sensing folding and metabolic stress as well as the unfolded protein response (UPR) (Malhotra and Kaufman 2007; Ron and Walter 2007; Lin et al. 2008), the heat-shock response (HSR) (Morimoto 2008; Shamovsky and Nudler 2008), $\mathrm{Ca}^{2+}$-sensing (Burdakov et al. 2005; Petersen et al. 2005), and inflammatory pathways (Medzhitov 2008; Zhang and Kaufman 2008). These are complemented by the components of the ubiquitinsumoylation proteasome system (UPS) found in the cytosol (Finley 2009), and lysosomal and autophagic degradation pathways that are also sensitive to multiple stress pathways ( $\mathrm{He}$ and Klionsky 2009). These signaling pathways maintain a healthful proteome by operating as integrated transcriptional/translation/folding systems to control the composition and capacity of the variable PN environments found in 


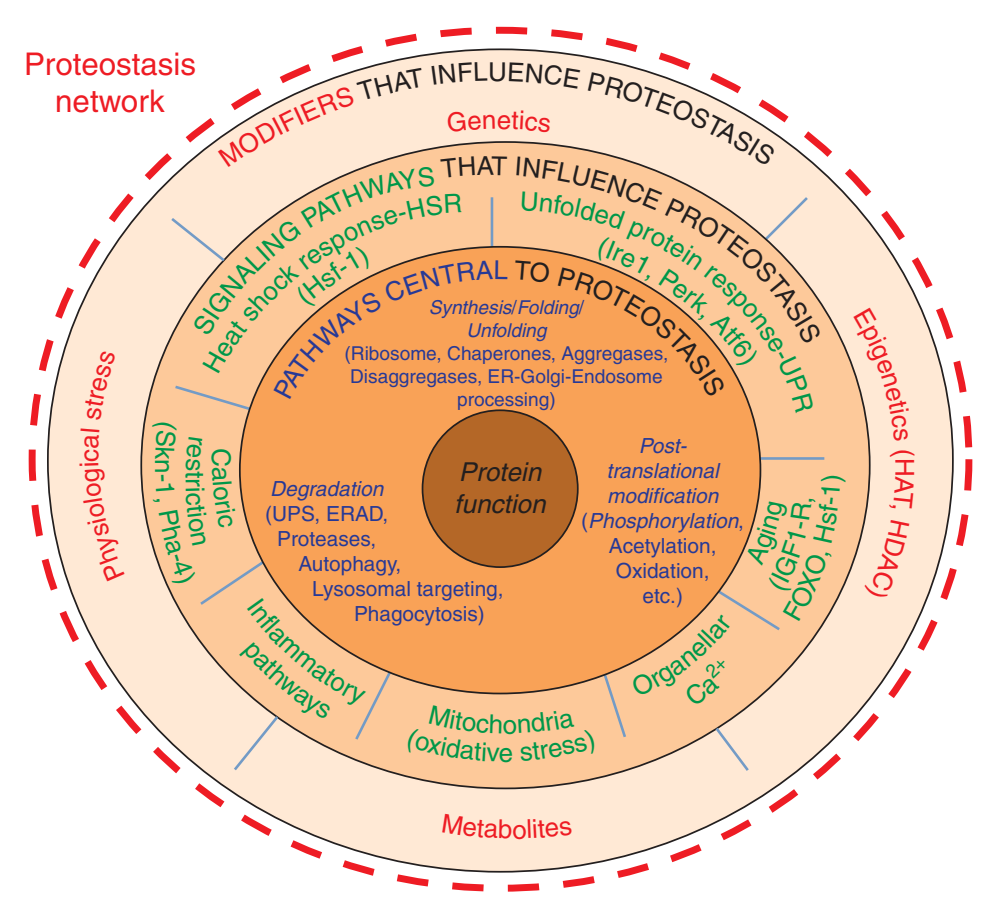

Figure 2. The Proteostasis Network. Shown are the interactions that comprise the PN, responsible for generating and maintaining the protein fold. Components comprising the PN outlined in the first layer (blue font) including the ribosome, Hsc70/Hsp70 and Hsp90 chaperones systems that direct folding, aggregases that promote aggregate formation, disaggregases that disassemble aggregates, as well as pathways that select proteins for degradation [e.g., the ubiquitin-proteasome system (UPS), endoplasmic reticulum (ER)-associated degradation (ERAD) systems, proteases, autophagic pathways, lysosomal/endosomal targeting pathways, and phagocytic pathways, the latter are responsible for the recognition, uptake, and degradation of extracellular proteins]. The second layer shows signaling pathways (green font) that influence the level and activity of components found in the first layer. The third layer (red font) includes genetic and epigenetic pathways, physiologic stressors, and intracellular metabolites that affect the activities defined by the second and first layer. Reproduced with permission from Elsevier Press (Fig. 2 in Powers et al. 2009).

different cell-types. They not only generate the protein fold but they protect it during youth and aging (Morimoto and Cuervo 2009; Powers et al. 2009).

The PN is necessarily ancient and coevolved with the remarkable diversity of peptide chain sequences and folds, reflecting the need to manage diverse patterns of folding energetics (Powers et al. 2009; Prahlad and Morimoto 2009; Hutt and Balch 2010). The PN very likely played an instrumental role in expanding the capacity of polypeptides to function in complex cell and tissue environments, and to perform increasingly precise cellular tasks associated with both cytosolic and membrane compartments found in human biology that ultimately requires complete integration of protein, cell, tissue, and organismal function (Hutt et al. 2009). Given the close coupling between the fold and the activity of the PN, any alterations in the polypeptide chain (e.g., a mutation, cotranslational and posttranslational modifications), or a change in the composition of the PN will lead to a change/breakdown in the folding network potentially triggering human pathologies. Thus, any effort to understand in vivo the impact of a mutation on folding and function will require careful consideration of the interdependence of folding energetics of the peptide chain and the PN in a given cell type. This of course will be tightly linked to the local tissue environment and organismal physiology that 
W.E. Balch et al.

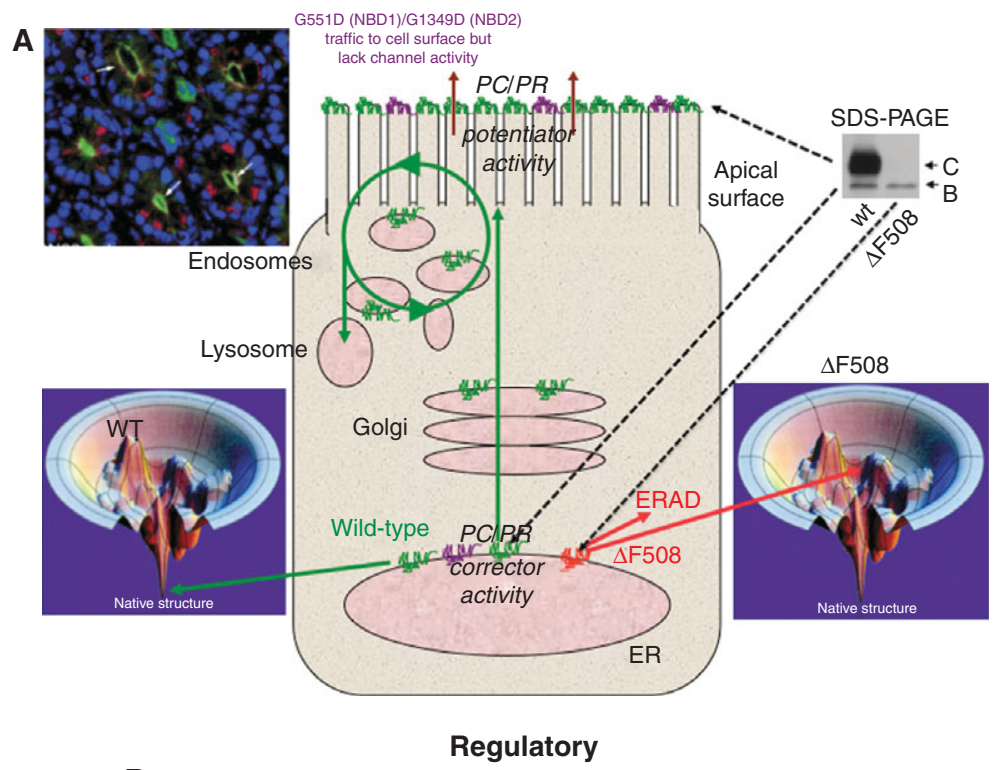

B

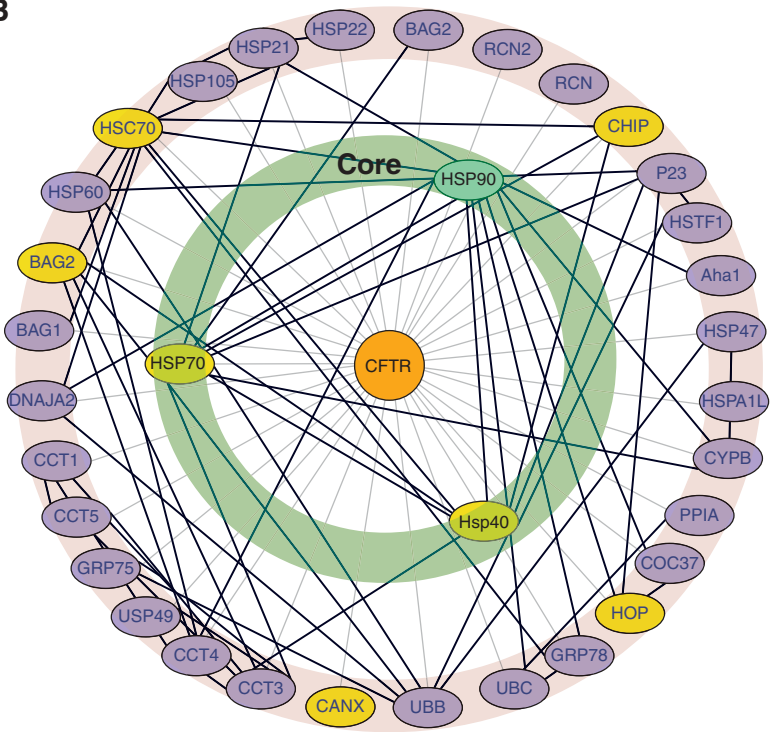

Figure 3. Cystic Fibrosis trafficking. (A) Illustrated is the trafficking itinerary of WT and three vCFTR $(\Delta \mathrm{F} 508$, G551E, G1349D) through the exocytic pathway. Wild-type CFTR folds efficiently as indicated by achieving its native state in the folding landscape (lower left) and indicated by the green icon in the endoplasmic reticulum (ER). It is trafficked to the apical surface of lung airway epithelial cells (upper left, green) where it has normal channel function. During transport through the Golgi, WT-CFTR is processed from the band B ER associated glycoform to the band C complex glycoform (upper right). In contrast, the $\Delta \mathrm{F} 508$ mutant is unstable in the ER (lower right) and as indicated by the red icon in the ER. It is efficiently degraded by ERAD. Typically, the only detectable form of $\Delta \mathrm{F} 508$ is the band B glycoform restricted to the ER prior to degradation. The G551E and G1349D mutants (purple) are folded and traffic normally to cell surface. However, both have gating defect preventing channel function. The G551E and G1349D vCFTR only require a potentiator to open the channel and restore function. In contrast, $\Delta \mathrm{F} 508$ requires the addition of a pharmacological chaperone (PC) corrector or alteration of the folding environment by a proteostasis regulator (PR) to restore delivery to the cell surface. $(B)$ The vCFTR folding interactome. Genes involved are indicated in capitals. (Wang et al. 2006). Reproduced with permission from Elsevier Press (Fig. 2B in Wang et al. 2006). (See facing page for legend.) 


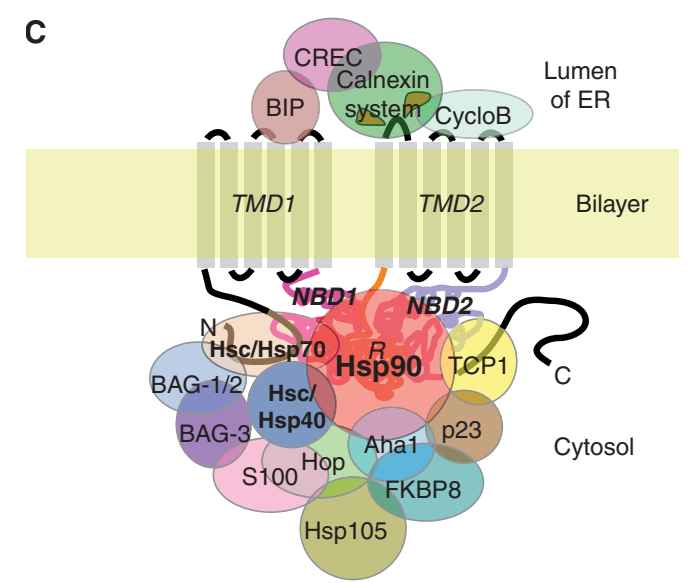

Figure 3. (Continued) (C) A key subset of PN factors that are now known to contribute to folding of vCFTR domains facing the cytosolic and the lumenal domain of the ER.

will likely trigger compensatory responses to deal with either the fold (Dill et al. 2008) and/ or the loss-of-function/gain-of-toxic function phenotype (Morimoto 2006; Morimoto and Cuervo 2009). The challenge will be to restore proteome balance (Hutt and Balch 2010).

To address the folding problem and its relationship to cell biology at a global level, we developed a general model, referred to as FoldEx, that describes, from the point of view of classic Michaelis-Menten kinetics, how the PN can influence the inherent energetics of the polypeptide chain folding kinetics to achieve transport of molecules such as CFTR through the exocytic pathway (Sekijima et al. 2005; Wiseman et al. 2007). This model was recently generalized to integrate folding energetics with the PN in all cellular compartments (Powers et al. 2009). The simple take-home from this general modeling approach (referred to as FoldFx), which provides a rigorous quantitative standard for biological folding, is that the local PN maintains a tight control on the folding energetics encoded by the primary peptide chain sequence to achieve biological function. Mutations such as those found in CF disease, which alter peptide chain folding energetics and its position relative to the inherent $\mathrm{PN}$ in the FoldEx and FoldFx models, predict that it
Proteostasis in Managing Cystic Fibrosis

will be necessary to alter PN function through biologic and/or pharmacologic means to restore proteome balance and function (Powers et al. 2009; Hutt and Balch 2010).

\section{CFTR BIOLOGY AND THE PN: WHAT GOES RIGHT AND WHAT GOES WRONG}

Diseases such as CF are not only a consequence of a deficiency in trafficking through the exocytic pathway, but in the function and stability of the protein at the cell surface reflecting recycling through the endocytic pathway and/or targeting for degradation by the lysosomal system (Guggino and Stanton 2006; Becq 2010; Okiyoneda et al. 2010) (Fig. 3A). Indeed, the existence of multiple trafficking compartments within the cell suggests that the cell operates in the context of multiple PNs that are unique to each compartment and individually subject to stress signaling regulation (Hutt et al. 2009). Moreover, transmembrane proteins such as CFTR are subject to folding challenges on two sides of the bilayer, the lumenal domain is subject to an unique series of oxidizing PNs found in the various trafficking compartments in which it transiently resides, whereas the cytosolic domain is sensitive to a reducing environment and a completely different set of $\mathrm{PN}$ components that remain in common throughout transit (Hutt et al. 2009).

WT CFTR is localized to the apical surface of epithelial cells where it regulates water and salt $\left(\mathrm{Na}^{+}\right.$and $\left.\mathrm{Cl}^{-}\right)$homeostasis to control the fluidity of the extracellular mucus layer critical for the function of multiple tissues including lung, intestine, and pancreas (Riordan 2008) (Fig. 3A). The 1480 amino acid polypeptide chain of CFTR is composed of two membrane-spanning domains (MSD1 and MSD2), each containing six transmembrane helices (TM), two nucleotide-binding domains (NBD1 and NBD2), and a cytoplasmic regulatory (R) domain. CFTR is synthesized by membranebound polyribosomes. Its nascent MSDs are imbedded in the bilayer of the endoplasmic reticulum (ER) where it acquires two N-linked glycans as part of the first step in the exocytic pathway (Fig. 3A). Despite its ER association, 
W.E. Balch et al.

CFTR folding requires extensive support from the cytosolic PN (Fig. 3B) to mediate proper folding of the cytosolic NBD1, R, and NBD2 domains with only small lumenal oriented loops connecting MSDs 1 and 2 contributing to the fold (Fig. 3C). The latter appear to be sensitive to the luminal chaperone systems. On proper folding, export from the ER to downstream compartments is managed by the COPII machinery (Wang et al. 2004; Wang et al. 2008), a critical rate-limiting event in disease (Wang et al. 2004). Following delivery to the Golgi compartment (Fig. 3A), the N-linked glycans undergo complex glycosylation preceding transfer to the cell surface where it is subject to regulation by endocytic pathways. Channel activity is regulated by a complex play of interactions involving kinases, phosphatases, and other binding partners that couple chloride transport with $\mathrm{Na}^{2+}$ transport (Guggino and Stanton 2006; Amaral and Kunzelmann 2007; Becq 2010).

We now appreciate that folding and maintenance of this multidomain protein as a functional chloride channel uses a sophisticated PN pathway (Fig. 3b,c) to promote not only proper domain assembly, but also interdomain assembly (Ollero et al. 2006; Wang et al. 2006; Wright et al. 2009). Molecular modeling approaches and cross-linking studies have shown that the NBD1 domain, synthesized immediately after MSD1, contacts the cytoplasmic loop (CL) 1 (MSD1) and 4 (MSD2) whereas NBD2, synthesized after MSD2, contacts CL2 (MSD1) and 3 (MSD2), interactions requiring substantial PN protection given the 5-10 minute time span required to synthesize full-length CFTR in vivo (He et al. 2008; Mornon et al. 2008; Rosser et al. 2008; Serohijos et al. 2008). Interestingly, in heterologous expression systems, even wildtype (WT) CFTR folding in the ER compartment is a very inefficient process with $>70 \%$ targeted for degradation by ER-associated degradation (ERAD) pathways, possibly reflecting differences in PN pathways. Whether this is true for endogenous expression in the context of the native PN found in human tissues remains controversial. One possibility is that cells present in an organismal tissue environment contain a different PN pool dictated by cell nonautonomous signaling pathways. In contrast, $>95 \%$ of $\Delta \mathrm{F} 508$ is retained in the ER in most cell/tissue types and targeted for degradation by ERAD, although some cell types are clearly more permissive for export of $\Delta \mathrm{F} 508$ than others, particularly heterologous cell culture expression systems, suggesting that the local PN strongly influences early folding events.

PN components facilitating folding of the cytosolic NBD1 and NBD2 are now recognized to include the Hsp40/Hsc-Hsp70 (Meacham et al. 1999; Meacham et al. 2001; Younger et al. 2004; Younger et al. 2006; Morito et al. 2008; Rosser et al. 2008; Grove et al. 2009) and Hsp90 chaperone systems (Loo et al. 1998; Fuller and Cuthbert 2000; Wang et al. 2006; Sun et al. 2008; Koulov et al. 2010). Calnexin, a membrane-anchored ER-associated chaperone, has been shown to interact with the immature forms of both WT and $\Delta$ F508 vCFTR (Pind et al. 1994), although its exact role in folding remains controversial (Zhang et al. 2001; Okiyoneda et al. 2002; Amaral 2004; Okiyoneda et al. 2004; Norez et al. 2006; Okiyoneda et al. 2008). In several studies, $\Delta \mathrm{F} 508$ is found to be associated more strongly with $\mathrm{Hsp} 40 / \mathrm{Hsc}-$ Hsp70 complexes than with WT, although these studies did not distinguish immature and mature pools in the case of WT. The peripheral (plasma membrane) reduce temperature rescued $\Delta$ F508 vCFTR pool is now appreciated to bind Hsc-Hsp70 suggesting that the PN influences CFTR folding and stability throughout its cellular itinerary (Hutt and Balch 2010; Okiyoneda et al. 2010). Moreover, the $\Delta$ F508 vCFTR-calnexin complex was found to have a longer half-life (Pind et al. 1994). One possibility that remains to be examined is that the misfolded $\Delta$ F508 vCFTR may be trapped in PN complex(es). Perhaps being unable to escape from a folding intermediate state, $\Delta$ F508 is targeted for degradation by the proteasome through the UPS pathways, potentially involving Hsp27 (Qu et al. 1997; Zhang et al. 2001; Youker et al. 2004; Sun et al. 2006; Wang et al. 2006; Zhang et al. 2006; Ahner et al. 2007; Vembar and Brodsky 2008). Recent studies suggest that it is the interaction of the PN 
component CHIP with Hsc-Hsp70 (Meacham et al. 2001) through the activity of the E3 ligase RMA1 (Grove et al. 2009) that promotes degradation. Intriguingly, siRNA silencing of the Hsp90 ATPase activator co-chaperone Ahal (Fig. 3C) in HEK293 cells and in primary airway cells homozygous for $\Delta \mathrm{F} 508$ resulted in stabilization, trafficking, and restoration of channel activity at the cell surface (Wang et al. 2006) (W. Balch and R. Frizzell, unpubl.). What is now very clear is that the primary defect in $\mathrm{CF}$ disease is an early PN-mediated folding event in the ER (Fig. 3b,c; see below Fig. 5) that requires further maintenance activity of the PN to prevent ERAD and targeting of cell surface rescued variant to the lysosomal pathway (Hutt and Balch 2010; Okiyoneda et al. 2010). Thus, the answer to the first question posed above is that WT and $\Delta$ F508 do interact with the $\mathrm{PN}$ differentially and that modulation of the PN function could contribute significantly to the treatment of CF by achieving proteome balance (Hutt et al. 2009; Hutt et al. 2010).

\section{CF THERAPEUTICS AND THE PN}

It should now be apparent that achieving correction of CF through the $\mathrm{PN}$ will be a multifaceted problem. Indeed, an extensive therapeutic pipeline has been developed by the CF foundation (http://www.cff.org/research/ DrugDevelopmentPipeline/) to address this challenge. Although this pipeline has been instrumental in moving the median survival age from 5-37 years old, it is largely a consequence of endpoint disease management at clinical centers using therapeutics that include digestive enzyme supplements, hypertonic $\mathrm{NaCl}$ inhalation protocols that facilitate increased lung function and mucociliary clearance, and, importantly, antibiotics that prevent recurrent bacterial infections that is the primary cause of premature death of CF patients (Proesmans et al. 2008; Frerichs and Smyth 2009; Jones and Helm 2009; Verkman and Galietta 2009; Becq 2010; Belcher and Vij 2010; Kreindler 2010; Zemanick et al. 2010). While no drugs to date are known to directly target the basic folding problem in disease, the quest for such drugs is in full force (Amaral and Kunzelmann 2007; Verkman and Galietta 2009; Becq 2010; Belcher and Vij 2010) with the goal of correcting the defects leading to the pathological triad (Fig. 1). Indeed, a large number of scaffolds have been identified that weakly influence the trafficking defect and/or channel activity. A number of these modulators can be found in the Cystic Fibrosis Foundation Therapeutics (CFFT) modulator library and are freely available to established and new investigators interested in pursuing CF.

Restoration of function through folding can occur through several mechanisms. One possibility is direct energetic stabilization of the fold through the use of therapeutics generally referred to as pharmacological chaperones (PCs) (Loo et al. 2008) (Fig. 4). "Corrector" PCs are thought to directly bind to the misfolded protein and provide additional stability as has been shown for G-protein coupled receptors (GPCRs) (Conn et al. 2007), Gaucher's (Yu et al. 2007; $\mathrm{Mu}$ et al. 2008), and transthyretin (TTR) mutants (Tojo et al. 2006; Hurshman Babbes et al. 2008). For example, compounds C3 and C4 from the CFFT modulator library have been suggested to mediate their activity through direct interaction with the CFTR protein (Loo and Clarke 2007; Wang et al. 2007; Loo et al. 2008; Grove et al. 2009). An alternative group of PCs used in the CF field are referred to as potentiators. CF potentiators promote surface channel activity but do not promote folding and/or trafficking of the nascent polypeptide. Importantly, the potentiator drug Vertex 770 (http://www.cff.org/research/ClinicalResearch/ FAQs/VX-770/) (Caputo et al. 2009; Van Goor et al. 2009; Wellhauser et al. 2009; Moran 2010) has recently completed a Phase II clinical trial with the G551D mutant (Fig. 3A), which is trafficked from the ER normally, cell surface localized but lacks channel activity. Treatment of patients leads to a significant increase in nasal potential difference, sweat chloride, and lung function, all hallmarks of restoration of lung function. Vertex 770 is thought to bind CFTR and favor channel open probability facilitating increased chloride flux (Riordan 2008), therefore artificially altering functionality, as do agonists 
W.E. Balch et al.
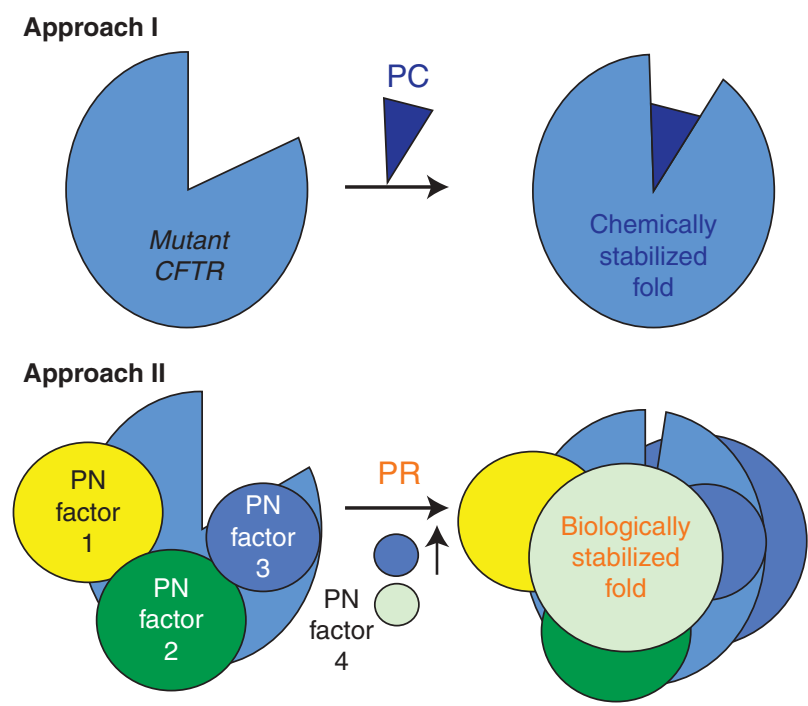

Figure 4. PCs and PRs. Illustrated are two approaches to target the folding problem in CF. PC is a pharmacological chaperone that can be a corrector or a potentiator by binding directly to the misfolded protein and energetically stabilizing the fold; PR is a proteostasis regulator that alters the level or content of PN factors (colored balls) that would improve the vCFTR fold.

and antagonists for GPCRs (Ulloa-Aguirre and Conn 2009).

In contrast to PCs, which may target specific steps in CFTR folding, a more encompassing approach may be achieved by using proteostasis regulators (PRs) (Balch et al. 2008) (Fig. 4), that is, molecules that adjust the composition and/ or concentration of the PN (Fig. 2) in a manner that favor $\Delta$ F508 vCFTR folding. PRs could be, in principle, similar to factors that stimulate stress responses leading to changes in $\mathrm{PN}$ composition of the cell that alleviate challenging misfolding problems. A PR that affects the cytosolic PN governing the folding of $\Delta$ F508 vCFTR could provide a more stabilizing environment, not only for ER export, but also for function at the cell surface. It is also possible that a combination of the various classes of PCs (correctors, potentiators) and PRs may synergize, as has been observed for Gaucher's disease in which rapid degradation in the ER could be reduced by $\mathrm{PR}$ modulation of the $\mathrm{PN}$, allowing sufficient protein to be produced to engage a PC corrector leading to an additional level of energetic stabilization either early or late in the trafficking pathway and rescue of the disease phenotype (Mu et al. 2008). Very recently, a potential PR (cystamine) that triggers autophagy pathways has been suggested as a potential mechanism to re-establish trafficking $\Delta$ F508 (Luciani et al. 2010). A lack of demonstration of functional channel activity at the surface limits the impact of such observations.

To date, the mechanism of action (MOA) of compounds identified from cell-based, high throughput screens (HTSs) designed to detect recovery of CFTR function at the cell surface are largely unknown. A case in point is Vertex 809 , a compound jointly developed by the CFFT and Vertex. Vertex 809, unlike Vertex 770 , is considered a corrector of the nascent polypeptide fold as it promotes exit from the ER (http://www.cff.org/treatments/Pipeline/). Thus, it could function either as a PC corrector or as a PR (Fig. 4). In a recent Phase II clinical trial with homozygous $\Delta \mathrm{F} 508$ patients, 809 had a modest, but encouraging capacity to correct nasal potential difference (http://www.vrtx. com/current-projects/drug-candidates/VX-809. html), although no effect on lung function was observed in the treatment regimens used to test potential efficacy. Given the modest 
improvement in channel function, the next study will proceed as a dual drug therapy by combining Vertex 809 with Vertex 770 (http://www.vrtx.com/current-projects/drugcandidates/vx-770.html), thereby providing a mechanism to further activate the channel function.

In general, ongoing efforts to identify CF therapeutics through biological analyses of PN function (e.g., Wang et al. 2006; Morito et al. 2008) strongly support the possibility that compounds that alter the folding energetics and/or PN biology of vCFTR have high potential for generating a more wild-type-like function, and hence, could have significant benefit in the clinic.

\section{MODEL SYSTEMS TO IDENTIFY PN THERAPEUTICS}

To study CF, we need both cell and animal model systems that accurately reproduce the operation of the PN in health and disease. With a strong focus on restoration of lung function, both primary WTand homozygous $\Delta \mathrm{F} 508$ $(\Delta \mathrm{F} / \Delta \mathrm{F})$ human bronchial epithelial (HBEs) $(\Delta \mathrm{F} / \Delta \mathrm{F}-\mathrm{HBEs})$ cells can be obtained from patient lungs during transplant and grown as epithelial monolayers on transwell dishes to assess trafficking, channel activity, and surface hydration at the air-liquid-surface (ALS) interface that lead to restoration of mucociliary function. These are routinely used by the $\mathrm{CF}$ community. At this juncture, human $\Delta \mathrm{F} /$ $\Delta$ F-HBEs are the gold standard for judging the efficacy of $\mathrm{CF}$ therapeutics coming through HTS pipeline for patient clinical trials. However, these are a challenging resource to generate and subject to the availability of tissue from CF patient lung transplants, although significant progress has been made in generating frozen stocks. It is important to note that HBEs derived from patients have very large differences in their channel activity and ability to respond to PCs and likely PRs, suggesting both the health of the lung at transplant and patient genetic and epigenetic (environmental) background has a major impact on the activity of the PN in maintaining vCFTR functionality.
Given the above limitation, the field has used a range of cell types including heterologous expression systems and a wide range of immortalized, tissue specific cell lines (lung, pancreas, and intestine) that can be readily maintained in culture. The key point is that each cell line and animal model (see below) likely reflect different PNs and therefore have limitations relative to providing insight into the pathophysiology of human disease. In most cases, the choice of the cell line used to define the role of the PN in CF disease is dictated by its availability, behavior (e.g., growth rate, formation of polarized monolayers, transfection efficiency, expression level of the exogenous gene, etc.), and strength of the endpoint assay that varies from biochemical assessment of trafficking patterns using SDS-PAGE to channel activity using Ussing chambers, fluorescence reporters and imaging, and patch-clamp recordings. In particular, use of polarized epithelial cells such as the lung CFBE41o- cell line expressing $\Delta$ F508 will likely be more physiologically relevant in terms of PN interactions than heterologous expression systems using fibroblast-derived cell lines. In this regard, the CFTR Folding Consortium (CFC) (http:// www.cftrfolding.org/) works with a series of model systems and has developed over 25 different assays that measure different features of CFTR trafficking and channel function. The CFC functions as a community resource to leverage this wide range of assays to generate the CFC "RoadMap" that attempts to measure the impact of a particular therapeutic on WT and vCFTR trafficking and function, with the goal of providing critical insight into MOA of newly discovered therapeutics.

To understand how studies in a particular cell line can impact our understanding of the PN in human disease, one can simply look at the half-life of the ER-restricted form of human $\Delta$ F508 vCFTR in a variety of cell lines. In general, $\Delta$ F508 half-life was found to be on the order of $1 \mathrm{~h}$ in Fischer rat thyroid (FRT), baby hamster kidney (BHK), monkey kidney (COS), and CFBE41o-human airway cell. In yeast, degradation is particularly notable and fasteven when WT CFTR fails to escape the ER, 
W.E. Balch et al.

suggesting a complete mismatch of CFTR folding requirements with the evolutionarily emergent properties of the yeast PN. Interestingly, and in contrast to the rather uniform short halflife of $\triangle \mathrm{F} 508 \mathrm{vCFTR}$ in the ER in a variety of cell lines, the mature form of $\Delta \mathrm{F} 508 \mathrm{vCFTR}$ that has trafficked to the cell surface showed a half-life of approximately $4 \mathrm{~h}$ in $\mathrm{CHO}$ cells compared to $1 \mathrm{~h}$ in CFBE41o- cells. It also showed altered endocytic recycling properties when comparing polarized and nonpolarized cell lines. This represents a significant difference in post-ER protein stability, possibly reflecting a difference in the PN environment and peripheral folding maintenance by the $\mathrm{PN}$ in these two cell types (Fig. 5) (Hutt and Balch 2010; Okiyoneda et al. 2010). Moreover, a recent comparison of 13 potentiators showed that three different mutants, including $\Delta$ F508 (NBD1, ER degraded, Fig. 3A), G551D (NBD1, cell surface localized, Fig. 3A), and G1349D (NBD2, cell surface localized) (Pedemonte et al. 2010), expressed in FRT cells or the human alveolar epithelial cells A549 responded to 10 of the 13 compounds tested, supporting the conclusion that these potentiators may be binding to vCFTR directly and their binding is not influenced by cellular environment (Pedemonte et al. 2010). Interestingly, when they examined the effect of known correctors on the aforementioned cells lines as well as on $\Delta F / \Delta F-H B E s$, they found that all but one of the correctors from the

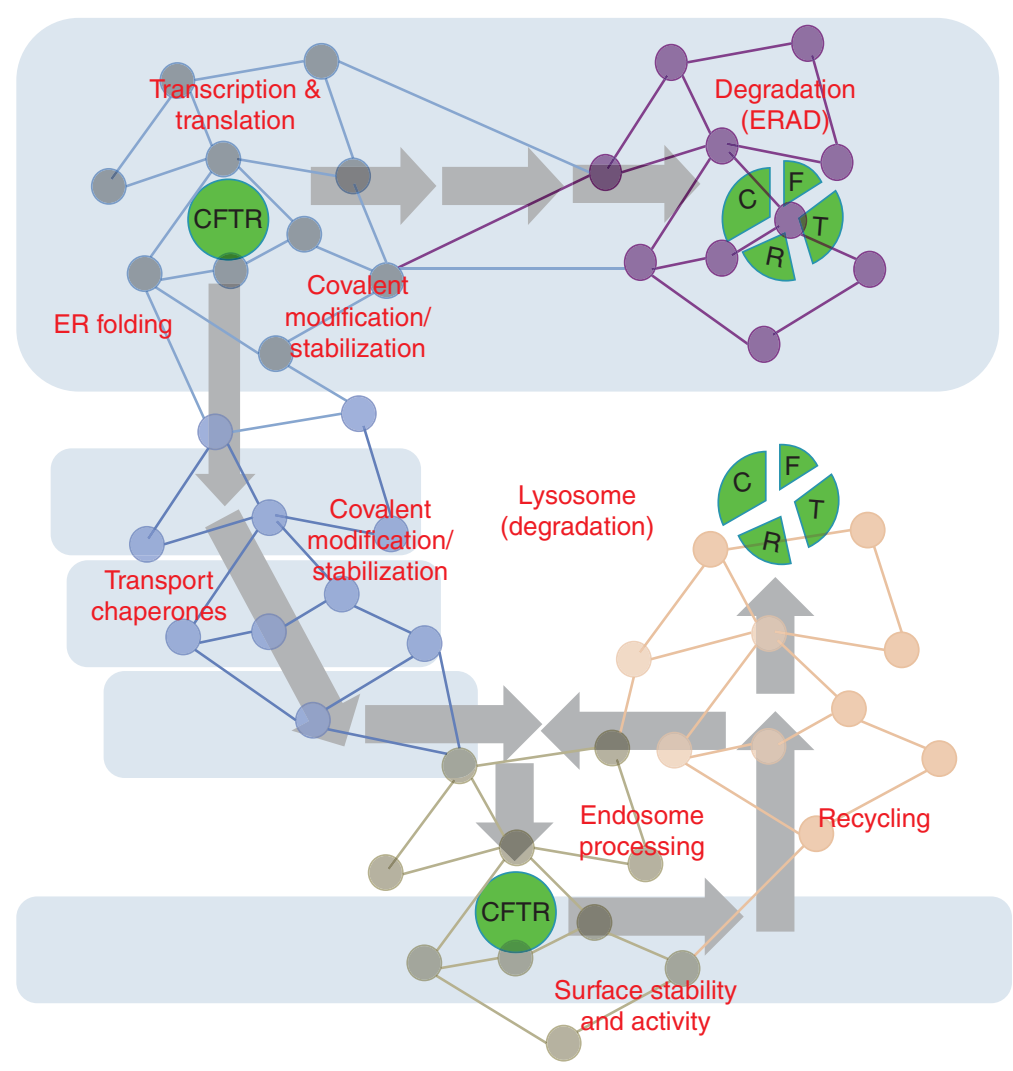

Figure 5. Management of vCFTR functions by the PN. A hypothetical view of the activity of subsets of factors present in the PN that function at the indicated step of the vCFTR folding and trafficking itinerary. Adjustments to one or more nodes through the activity of PRs cannot only restore CFTR function, but re-establish connectivity to the biological network of the cell supporting normal physiology, thus alleviating the pathologic CF triad contributing to onset and progression of disease (Fig. 1). 
CFFT modulator library were effective in only one cell line, however, C3 and C4, shown to act directly on vCFTR, were effective in all three cell lines (Pedemonte et al. 2010). Thus, correction by a PC or PR appears very sensitive to the $\mathrm{PN}$, emphasizing the importance of model systems to accurately recapitulate the human tissue environment.

To address the organismal respond to therapeutics, a number of mouse models have been generated to better understand the physiological complications associated with the intact diseased tissue. Strikingly, all of the CF mice models fail to recapitulate respiratory defects associated with human CF and therefore fail to manifest inflammatory markers. Moreover, they lack susceptibility to bacterial infection and do not recapitulate pancreatic and hepatobiliary phenotypes found in the human condition (Scholte et al. 2004). However, they do have defects in nasal potential and develop prominent intestinal obstruction, resulting in peritonitis and nutritional insufficiency typically seen in CF patients. A number of reasons could account for the observed differences between mouse and human pathophysiology. They include variations in airway biology and/or the expression of alternative, cAMPstimulated, nonCFTR chloride channels in the murine lung. In addition to these physiological and cellular differences, the amino acid sequence of the mouse CFTR shows only $88 \%$ similarilty and $78 \%$ identity to human CFTR suggesting possible differences in the structurefunction relationship of these proteins with their respective handling by the evolutionaryand environmental triggered emergent properties of the mouse and human PNs (Liu et al. 2007).

The recent development of the CF pig model is a very exciting addition to the CF field in that the pig phenocopies human lung and intestinal disease (Rogers et al. 2008; Stoltz et al. 2010; Wine 2010). In addition to striking similarities in porcine and human lung physiology, a sequence alignment between pCFTR and hCFTR reveals that the two proteins share 92\% identity, suggesting that the proteins may have similar folding properties that would require a similar level of involvement of conserved PN components for folding. In fact, single channel recordings of the two proteins revealed very similar unitary channel conductance (6.7 pS for hCFTR vs. $8.3 \mathrm{pS}$ pCFTR) as well as identical open probability $\left(\mathrm{P}_{\mathrm{o}}=0.39\right)$ (Rogers et al. 2008). Additionally, it was found that, in isolated airway epithelia from porcine trachea, pCFTR showed a similar amiloride sensitive sodium current to that seen in human airway epithelial cells. A close examination of trafficking of WT and mutant $\Delta$ F508 CFTR in human cells revealed that although both WT pCFTR and hCFTR are similarly delivered to the cell surface, the $\Delta$ F508 mutant of pCFTR shows a significant level of escape from the typical ER retention resulting in significant localization to the apical membrane. In addition, the $\mathrm{p} \Delta \mathrm{F} 508$-CFTR showed a higher $\mathrm{P}_{\mathrm{o}}$ and residual basal current as compared to the same mutation in hCFTR (Rogers et al. 2008; Stoltz et al. 2010). These differences could in part be attributed to the sequence differences between porcine and human CFTR. In general, the pig lung recapitulates the CF-triad pathology (Fig. 1). However, differences may also exist between the PN of the porcine and human lung cells, which could contribute to onset and intensity of pathophysiology. In general, current evidence suggests that the CF pig may provide a good model of CF lung disease allowing for a better understanding of the emergent properties of the PN in disease (Stoltz et al. 2010; Wine 2010). It will likely serve as a good experimental model for the testing of novel therapeutics aimed at treating the basic defect in the human disease.

In summary, the answer to question 2 posed above (What is the value of models in assessing $\mathrm{PN}$ function in restoration of vCFTR?) is that although the primary defect observed in humans (e.g., failure to fold and exit the ER) is preserved in all models tested, PN pathways that promote restoration of function may be more cell type and species specific, reflecting the composition and regulation of the $\mathrm{PN}$ that must integrate the vCFTR fold with many other aspects of cellular function found in different cell and organismal states. 
W.E. Balch et al.

\section{CHARACTERIZATION OF NEW TARGETS AFFECTING RESTORATION OF TISSUE FUNCTION}

Although phenotypic rescue of disease through the PN by use of small molecules that affect CFTR folding is an important goal of the CF drug development pipeline, alternative strategies are now evident including directly targeting $\mathrm{Na}^{++}$-channel regulation that is defective in the absence of CFTR (Boucher 2007; Donaldson and Boucher 2007; Lazarowski and Boucher 2009), up-regulation of alternative $\mathrm{Ca}^{2+}$-activated $\mathrm{Cl}^{-}$channels (CaCC) to replace vCFTR loss, development of anti-inflammatory strategies to counter the hyperactive inflammatory state found in response to the absence of WT CFTR and in the diseased CF lung, and/ or provision of mucolytic agents to improve clearance and reduced pathogenesis (e.g., the CF triad (Fig. 1) (Proesmans et al. 2008; Frerichs and Smyth 2009; Jones and Helm 2009; Verkman and Galietta 2009; Becq 2010; Belcher and Vij 2010; Kreindler 2010; Zemanick et al. 2010).

Although each of the above therapeutic approaches would be expected to provide endpoint benefit, known drugs do not solve the folding problem associated with loss of CFTRmisfolding, loss of trafficking, and degradation in the ER. Thus, an important goal is to understand the MOA of drugs obtained from cellbased screens that could function through PN modulators that would solve this problem. In this regard, an extensive effort has been devoted to the development of a CF "interaction" network through data-mining efforts provided by GeneGo (CF MetaMiner [CF]) (Wright et al. 2009) that provides a carefully annotated, systems level view of components contributing to CFTR function and correction of disease etiology. Moreover, in addition to studies on the WT and $\Delta$ F508-CFTR interactome (Wang et al. 2006), a series of bioinformatic studies have analyzed both the genomic and whole cell proteomic responses of human tissue to disease (Lipecka et al. 2006; Ollero et al. 2006; Pollard et al. 2006; Singh et al. 2006; Singh et al. 2008). Together, these studies illuminate an extensive network of interactions that will need to be restored to obtain full correction of the disease phenotype (Fig. 5). All of these pathways are heavily impacted by the PN (Powers et al. 2009).

In contrast to bioinformatic approaches based on the current extensive CF literature (e.g., GeneGo), genomic approaches (e.g., microarrays) report indirectly on CFTR function by monitoring cellular mRNA pools. They report on changes in stress response pathways reflecting PN modulation in response to disease and correction. In contrast, proteomic studies based on mass spectrometry report the level of recovery of known interactors in response to disease and correction of the fold (Wang et al. 2006) (W. Balch and J. Yates, unpubl.). The CFC (http://www.cftrfolding.org/) has used both genomic and proteomic data as well as the GeneGo MetaMiner database to highlight a series of gene products, referred to as the "top 10" that include a number of PN components, that are likely candidates for corrective action in CF disease (W. Skach and R. Frizzell, pers. comm.). The ability to identify key players involved in restoration of CFTR function emphasizes the importance of further studies that seek to better understand the individual contribution of these proteins in CFTR folding, trafficking, and function, and the development of reagents that directly target these players to influence restoration of vCFTR function (Fig. 5). For example, screens against key regulators of the UPR, heat shock, and other PN regulatory pathways may yield a wealth of therapeutics that manipulate the $\mathrm{PN}$ in a fashion that is favorable for correction of vCFTR. Moreover, these compounds could differentially affect the various disease causing mutants depending on the folding step that is disrupted and the local PN capacity (Powers et al. 2009; Hutt and Balch 2010). In this regard, correction through the $P N$ could provide a personalized approach to correction of CF disease (Becq 2010). The value of targeting the $\mathrm{PN}$ is that therapeutically corrective environments would target the unique energetics of the fold influenced by different mutations and the local folding environment of a patient.

The above studies directly address question three posed earlier: Do we need to focus on 
additional targets/pathways to generate maximum therapeutic benefit? The answer to this question is a definitive yes, even if only to provide further endpoint benefit to the pathophysiology observed in the clinic.

\section{EMERGENT PROPERTIES OF THE PN AS A GUIDE FOR RESCUE OF MISFOLDING DISEASE}

An overview of the concepts critical for the development of $\mathrm{PN}$-based therapeutics as a corrective solution for $\mathrm{CF}$ and the experimental approaches that will ultimately impact these efforts has been outlined above. In principle, correcting the vCFTR fold could significantly restore the underlying defect (e.g., yield a WT interactome) that could result in "self-correction" of the pathology triad (Fig. 5) typically observed in disease in response to a gain of "normal" channel function. This again focuses on question 3: Are there limits to what we can correct? At the moment there is not a definitive answer. At one level, a combination of at least two PN therapeutics, one that targets the primary defect, folding, and export from the ER, and a second that targets cell surface stability and/or channel gating would be predicted to have significant benefit and would serve as a starting point. This hypothesis is being tested in the combined Vertex 809/Vertex 770 phase II clinical trial mentioned above, although in the absence of an understanding of the role of 809 in correction, Vertex 770 may not be the best approach (http://www.vrtx.com/currentprojects/drug-candidates/VX-809.html). Until we more fully understand the role of PN biology in CFTR and vCFTR function, we may have to move more aggressively to assemble the various operating parts of the $\mathrm{PN}$ to understand what a truly effective therapeutic strategy needs to be for correction.

Given the above, it is anticipated that PNbased therapeutics would work most effectively when started early and would, in principle, require administration throughout life to maintain function. However, the vast majority of CF patients already have seriously compromised organ systems, particularly the lung, given its continuous exposure to pathogens. How much corrective activity would be required from PN modulators to achieve efficacy in late stage disease remains an open question. Indeed, until we more completely understand the PN-based folding problem, we will continue to require additional strategies that focus on non-CFTR targets to maximize patient benefit (question 4 posed above). At the minimum, the hope would be that therapeutics that augment the function of the PN would arrest disease progression so that the disease could be more effectively managed through the plethora of therapeutics that target endpoint pathophysiology (Fig. 1) that are now available in the CF pipeline (http://www.cff.org/research/DrugDevelopmentPipeline/). Interestingly, it is well known that epithelial cells in most organ systems routinely undergo turnover, particularly in response to damage as occurs in the CF airway; thus, there remains the potential for correction through epigenetic mechanisms that may teach the cell to more effectively manage the $\Delta$ F508 pathophysiological phenotype (Hutt et al. 2010). Moreover, it is now recognized that worm and mouse PN environments can be readily manipulated to protect against folding disorders and aging through the modulation of aging, diet, and oxidative pathways (Cohen et al. 2006; Cohen et al. 2009; Douglas and Dillin 2010; Mair et al. 2009; Panowski and Dillin 2009). It is therefore not unreasonable to suggest that similar approaches may be applicable to CF and a broad range of folding disorders.

\section{REFERENCES}

Ahner A, Nakatsukasa K, Zhang H, Frizzell RA, Brodsky JL. 2007. Small heat-shock proteins select deltaF508-CFTR for endoplasmic reticulum-associated degradation. $\mathrm{Mol}$ Biol Cell 18: 806-814.

Amaral MD. 2004. CFTR and chaperones: processing and degradation. J Mol Neurosci 23: 41-48.

Amaral MD, Kunzelmann K. 2007. Molecular targeting of CFTR as a therapeutic approach to cystic fibrosis. Trends Pharmacol Sci 28: 334-341.

Balch WE, Morimoto RI, Dillin A, Kelly JW. 2008. Adapting proteostasis for disease intervention. Science 319: $916-$ 919. 
W.E. Balch et al.

Becq F. 2010. Cystic fibrosis transmembrane conductance regulator modulators for personalized drug treatment of cystic fibrosis: progress to date. Drugs 70: $241-259$.

Belcher CN, Vij N. 2010. Protein processing and inflammatory signaling in Cystic Fibrosis: challenges and therapeutic strategies. Curr Mol Med 10: 82-94.

Boucher RC. 2007. Evidence for airway surface dehydration as the initiating event in CF airway disease. J Intern Med 261: $5-16$.

Burdakov D, Petersen OH, Verkhratsky A. 2005. Intraluminal calcium as a primary regulator of endoplasmic reticulum function. Cell Calcium 38: 303-310.

Caputo A, Hinzpeter A, Caci E, Pedemonte N, Arous N, Di Duca M, Zegarra-Moran O, Fanen P, Galietta LJ. 2009. Mutation-specific potency and efficacy of cystic fibrosis transmembrane conductance regulator chloride channel potentiators. J Pharmacol Exp Ther 330: 783-791.

Cohen E, Bieschke J, Perciavalle RM, Kelly JW, Dillin A. 2006. Opposing activities protect against age-onset proteotoxicity. Science 313: 1604-1610.

Cohen E, Paulsson JF, Blinder P, Burstyn-Cohen T, Du D, Estepa G, Adame A, Pham HM, Holzenberger M, Kelly JW, et al. 2009. Reduced IGF-1 signaling delays ageassociated proteotoxicity in mice. Cell 139: 1157-1169.

Collaco JM, Cutting GR. 2008. Update on gene modifiers in cystic fibrosis. Curr Opin Pulm Med 14: 559-566.

Conn PM, Ulloa-Aguirre A, Ito J, Janovick JA. 2007. G protein-coupled receptor trafficking in health and disease: lessons learned to prepare for therapeutic mutant rescue in vivo. Pharmacol Rev 59: 225-250.

Dill KA, Ozkan SB, Shell MS, Weikl TR. 2008. The protein folding problem. Annu Rev Biophys 37: 289-316.

Donaldson SH, Boucher RC. 2007. Sodium channels and cystic fibrosis. Chest 132: 1631-1636.

Douglas PM, Dillin A. 2010. Protein homeostasis and aging in neurodegeneration. J Cell Biol 190: 719-729.

Finley D. 2009. Recognition and processing of ubiquitinprotein conjugates by the proteasome. Annu Rev Biochem 78: $477-513$.

Frerichs C, Smyth A. 2009. Treatment strategies for cystic fibrosis: what's in the pipeline? Expert Opin Pharmacother 10: $1191-1202$.

Fuller W, Cuthbert AW. 2000. Post-translational disruption of the delta F508 cystic fibrosis transmembrane conductance regulator (CFTR)-molecular chaperone complex with geldanamycin stabilizes delta F508 CFTR in the rabbit reticulocyte lysate. J Biol Chem 275: 37462-37468.

Grove DE, Rosser MF, Ren HY, Naren AP, Cyr DM. 2009. Mechanisms for Rescue of Correctable Folding Defects in CFTR\{Delta\}F508. Mol Biol Cell 20: 4059-4069.

Guggino WB, Stanton BA. 2006. New insights into cystic fibrosis: molecular switches that regulate CFTR. Nat Rev Mol Cell Biol 7: 426-436.

He C, Klionsky DJ. 2009. Regulation mechanisms and signaling pathways of autophagy. Annu Rev Genet 43: 67-93.

He L, Aleksandrov AA, Serohijos AW, Hegedus T, Aleksandrov LA, Cui L, Dokholyan NV, Riordan JR. 2008. Multiple membrane-cytoplasmic domain contacts in the cystic fibrosis transmembrane conductance regulator (CFTR) mediate regulation of channel gating. J Biol Chem 283: 26383-26390.

Hurshman Babbes AR, Powers ET, Kelly JW. 2008. Quantification of the thermodynamically linked quaternary and tertiary structural stabilities of transthyretin and its disease-associated variants: the relationship between stability and amyloidosis. Biochemistry 47: 6969-6984.

Hutt D, Balch WE. 2010. Cell Biology. The proteome in balance. Science 329: 766-767.

Hutt DM, Powers ET, Balch WE. 2009. The proteostasis boundary in misfolding diseases of membrane traffic. FEBS Lett 583: 2639-2646.

Hutt DH, Herman D, Rodrigues APC, Noel S, Pilewski JM, Matteson J, Hoch B, Wellner W, Kelly JW, Schmidt A, et al. 2010. Reduced histone deacetylase 7 activity restores function to misfolded CFTR in cystic fibrosis. Nat Chem Biol 6: 25-33.

Jones AM, Helm JM. 2009. Emerging treatments in cystic fibrosis. Drugs 69: 1903-1910.

Koulov AV, Lapointe P, Lu B, Razvi A, Coppinger J, Dong MQ, Matteson J, Laister R, Arrowsmith C, Yates JR, et al. 2010. Biological and Structural Basis for Ahal Regulation of Hsp90 ATPase Activity in Maintaining Proteostasis in the Human Disease Cystic Fibrosis. Mol Biol Cell 21: 871-874.

Kreindler JL. 2010. Cystic fibrosis: exploiting its genetic basis in the hunt for new therapies. Pharmacol Ther 125: 219-229.

Lazarowski ER, Boucher RC. 2009. Purinergic receptors in airway epithelia. Curr Opin Pharmacol 9: 262-267.

Lin JH, Walter P, Yen TS. 2008. Endoplasmic reticulum stress in disease pathogenesis. Annu Rev Pathol 3: 399-425.

Lipecka J, Norez C, Bensalem N, Baudouin-Legros M, Planelles G, Becq F, Edelman A, Davezac N. 2006. Rescue of DeltaF508-CFTR (cystic fibrosis transmembrane conductance regulator) by curcumin: involvement of the keratin 18 network. J Pharmacol Exp Ther 317: 500-505.

Liu X, Luo M, Zhang L, Ding W, Yan Z, Engelhardt JF. 2007. Bioelectric properties of chloride channels in human, pig, ferret, and mouse airway epithelia. Am J Respir Cell Mol Biol 36: 313-323.

Loo TW, Clarke DM. 2007. Chemical and pharmacological chaperones as new therapeutic agents. Expert Rev Mol Med 9: 1-18.

Loo TW, Bartlett MC, Clarke DM. 2008. Correctors promote folding of the CFTR in the endoplasmic reticulum. Biochem J 413: 29-36.

Loo MA, Jensen TJ, Cui L, Hou Y, Chang XB, Riordan JR. 1998. Perturbation of Hsp90 interaction with nascent CFTR prevents its maturation and accelerates its degradation by the proteasome. Embo J 17: 6879-6887.

Luciani A, Villella VR, Esposito S, Brunetti-Pierri N, Medina D, Settembre C, Gavina M, Pulze L, Giardino I, Pettoello-Mantovani M, et al. 2010. Defective CFTR induces aggresome formation and lung inflammation in cystic fibrosis through ROS-mediated autophagy inhibition. Nat Cell Biol 12: 863-875.

Mair W, Panowski SH, Shaw RJ, Dillin A. 2009. Optimizing dietary restriction for genetic epistasis analysis and gene discovery in C. elegans. PLoS One 4: e4535. 
Malhotra JD, Kaufman RJ. 2007. The endoplasmic reticulum and the unfolded protein response. Semin Cell Dev Biol 18: 716-731.

Meacham GC, Lu Z, King S, Sorscher E, Tousson A, Cyr DM. 1999. The Hdj-2/Hsc70 chaperone pair facilitates early steps in CFTR biogenesis. EMBO J 18: 1492-1505.

Meacham GC, Patterson C, Zhang W, Younger JM, Cyr DM. 2001. The Hsc70 co-chaperone CHIP targets immature CFTR for proteasomal degradation. Nat Cell Biol 3: $100-105$.

Medzhitov R. 2008. Origin and physiological roles of inflammation. Nature 454: 428-435.

Moran O. 2010. Model of the cAMP activation of chloride transport by CFTR channel and the mechanism of potentiators. J Theor Biol 262: 73-79.

Morimoto RI. 2006. Stress, aging, and neurodegenerative disease. N Engl J Med 355: 2254-2255.

Morimoto RI. 2008. Proteotoxic stress and inducible chaperone networks in neurodegenerative disease and aging. Genes Dev 22: 1427-1438.

Morimoto RI, Cuervo AM. 2009. Protein homeostasis and aging: taking care of proteins from the cradle to the grave. J Gerontol (A Biol Sci Med Sci) 64: 167-170.

Morito D, Hirao K, Oda Y, Hosokawa N, Tokunaga F, Cyr DM, Tanaka K, Iwai K, Nagata K. 2008. Gp78 cooperates with RMA1 in endoplasmic reticulum-associated degradation of CFTRDeltaF508. Mol Biol Cell 19: 1328-1336.

Mornon JP, Lehn P, Callebaut I. 2008. Atomic model of human cystic fibrosis transmembrane conductance regulator: membrane-spanning domains and coupling interfaces. Cell Mol Life Sci 65: 2594-2612.

Mu TW, Ong DS, Wang YJ, Balch WE, Yates JR III, Segatori L, Kelly JW. 2008. Chemical and biological approaches synergize to ameliorate protein-folding diseases. Cell 134: $769-781$.

Norez C, Noel S, Wilke M, Bijvelds M, Jorna H, Melin P, DeJonge H, Becq F. 2006. Rescue of functional delF508CFTR channels in cystic fibrosis epithelial cells by the alpha-glucosidase inhibitor miglustat. FEBS Lett 580: 2081-2086.

Okiyoneda T, Barriere H, Bagdany M, Rabeh WM, Du K, Hohfeld J, Young JC, Lukacs GL. 2010. Peripheral protein quality control removes unfolded CFTR from the plasma membrane. Science 329: 805-810.

Okiyoneda T, Harada K, Takeya M, Yamahira K, Wada I, Shuto T, Suico MA, Hashimoto Y, Kai H. 2004. Delta F508 CFTR pool in the endoplasmic reticulum is increased by calnexin overexpression. Mol Biol Cell 15: 563-574.

Okiyoneda T, Niibori A, Harada K, Kohno T, Michalak M, Duszyk M, Wada I, Ikawa M, Shuto T, Suico MA, et al. 2008. Role of calnexin in the ER quality control and productive folding of CFTR; differential effect of calnexin knockout on wild-type and DeltaF508 CFTR. Biochim Biophys Acta 1783: 1585-1594.

Okiyoneda T, Wada I, Jono H, Shuto T, Yoshitake K, Nakano N, Nagayama S, Harada K, Isohama Y, Miyata T, et al. 2002. Calnexin Delta $185-520$ partially reverses the misprocessing of the Delta F508 cystic fibrosis transmembrane conductance regulator. FEBS Lett 526: 87-92.
Ollero M, Brouillard F, Edelman A. 2006. Cystic fibrosis enters the proteomics scene: new answers to old questions. Proteomics 6: 4084-4099.

Panowski SH, Dillin A. 2009. Signals of youth: endocrine regulation of aging in Caenorhabditis elegans. Trends Endocrinol Metab 20: 259-264.

Pedemonte N, Tomati V, Sondo E, Galietta LJ. 2010. Influence of cell background on pharmacological rescue of mutant CFTR. Am J Physiol Cell Physiol 298: C866-C874.

Petersen OH, Michalak M, Verkhratsky A. 2005. Calcium signalling: past, present and future. Cell Calcium 38: $161-169$.

Pind S, Riordan JR, Williams DB. 1994. Participation of the endoplasmic reticulum chaperone calnexin (p88, IP90) in the biogenesis of the cystic fibrosis transmembrane conductance regulator. J Biol Chem 269: 12784-12788.

Pollard HB, Eidelman O, Jozwik C, Huang W, Srivastava M, Ji XD, McGowan B, Norris CF, Todo T, Darling T, et al. 2006. De novo biosynthetic profiling of high abundance proteins in cystic fibrosis lung epithelial cells. Mol Cell Proteomics 5: 1628-1637.

Powers ET, Morimoto RI, Dillin A, Kelly JW, Balch WE. 2009. Biological and Chemical Approaches to Diseases of Proteostasis Deficiency. Annu Rev Biochem (In press).

Prahlad V, Morimoto RI. 2009. Integrating the stress response: lessons for neurodegenerative diseases from C. elegans. Trends Cell Biol 19: 52-61.

Proesmans M, Vermeulen F, De Boeck K. 2008. What's new in cystic fibrosis? From treating symptoms to correction of the basic defect. Eur J Pediatr 167: 839-849.

Qu BH, Strickland E, Thomas PJ. 1997. Cystic fibrosis: a disease of altered protein folding. J Bioenerg Biomembr 29: 483-490.

Riordan JR. 2008. CFTR function and prospects for therapy. Annu Rev Biochem 77: 701-726.

Rogers CS, Stoltz DA, Meyerholz DK, Ostedgaard LS, Rokhlina T, Taft PJ, Rogan MP, Pezzulo AA, Karp PH, Itani OA, et al. 2008. Disruption of the CFTR gene produces a model of cystic fibrosis in newborn pigs. Science 321: 1837-1841.

Ron D, Walter P. 2007. Signal integration in the endoplasmic reticulum unfolded protein response. Nat Rev Mol Cell Biol 8: 519-529.

Rosser MF, Grove DE, Chen L, Cyr DM. 2008. Assembly and misassembly of cystic fibrosis transmembrane conductance regulator: folding defects caused by deletion of F508 occur before and after the calnexin-dependent association of membrane spanning domain (MSD) 1 and MSD2. Mol Biol Cell 19: 4570-4579.

Scholte BJ, Davidson DJ, Wilke M, De Jonge HR. 2004. Animal models of cystic fibrosis. J Cyst Fibros 3: 183-190.

Sekijima Y, Wiseman RL, Matteson J, Hammarstrom P, Miller SR, Sawkar AR, Balch WE, Kelly JW. 2005. The biological and chemical basis for tissue-selective amyloid disease. Cell 121: 73-85.

Serohijos AW, Hegedus T, Aleksandrov AA, He L, Cui L, Dokholyan NV, Riordan JR. 2008. Phenylalanine-508 mediates a cytoplasmic-membrane domain contact in the CFTR 3D structure crucial to assembly and channel function. Proc Natl Acad Sci 105: 3256-3261. 
W.E. Balch et al.

Shamovsky I, Nudler E. 2008. New insights into the mechanism of heat shock response activation. Cell Mol Life Sci 65: 855-861.

Singh OV, Pollard HB, Zeitlin PL. 2008. Chemical rescue of deltaF508-CFTR mimics genetic repair in cystic fibrosis bronchial epithelial cells. Mol Cell Proteomics 7: 10991110.

Singh OV, Vij N, Mogayzel PJ Jr, Jozwik C, Pollard HB, Zeitlin PL. 2006. Pharmacoproteomics of 4-phenylbutyrate-treated IB3-1 cystic fibrosis bronchial epithelial cells. J Proteome Res 5: 562-571.

Stoltz DA, Meyerholz DK, Welsh MJ. 2010. Cystic fibrosis pigs deelop lung disease and exhibit defective bacterial eradication at birth. Sci Transl Med 2: 29.

Sun F, Mi Z, Condliffe SB, Bertrand CA, Gong X, Lu X, Zhang R, Latoche JD, Pilewski JM, Robbins PD, et al. 2008. Chaperone displacement from mutant cystic fibrosis transmembrane conductance regulator restores its function in human airway epithelia. FASEB $J$ 22: 3255-3263.

Sun F, Zhang R, Gong X, Geng X, Drain PF, Frizzell RA. 2006. Derlin-1 promotes the efficient degradation of the cystic fibrosis transmembrane conductance regulator (CFTR) and CFTR folding mutants. J Biol Chem 281: 36856-36863.

Tojo K, Sekijima Y, Kelly JW, Ikeda S. 2006. Diflunisal stabilizes familial amyloid polyneuropathy-associated transthyretin variant tetramers in serum against dissociation required for amyloidogenesis. Neurosci Res 56: 441-449.

Ulloa-Aguirre A, Conn PM. 2009. Targeting of G proteincoupled receptors to the plasma membrane in health and disease. Front Biosci 14: 973-994.

Van Goor F, Hadida S, Grootenhuis PD, Burton B, Cao D, Neuberger T, Turnbull A, Singh A, Joubran J, Hazlewood A, et al. 2009. Rescue of CF airway epithelial cell function in vitro by a CFTR potentiator, VX-770. Proc Natl Acad Sci 106: $18825-18830$.

Vembar SS, Brodsky JL. 2008. One step at a time: endoplasmic reticulum-associated degradation. Nat Rev Mol Cell Biol 9: 944-957.

Verkman AS, Galietta LJ. 2009. Chloride channels as drug targets. Nat Rev Drug Discov 8: 153-171.

Wang X, Koulov AV, Kellner WA, Riordan JR, Balch WE. 2008. Chemical and biological folding contribute to temperature-sensitive DeltaF508 CFTR trafficking. Traffic 9: 1878-1893.

Wang Y, Loo TW, Bartlett MC, Clarke DM. 2007. Correctors promote maturation of cystic fibrosis transmembrane conductance regulator (CFTR)-processing mutants by binding to the protein. J Biol Chem 282: 33247-33251.

Wang X, Matteson J, An Y, Moyer B, Yoo JS, Bannykh S, Wilson IA, Riordan JR, Balch WE. 2004. COPIIdependent export of cystic fibrosis transmembrane conductance regulator from the ER uses a di-acidic exit code. J Cell Biol 167: 65-74.
Wang X, Venable J, LaPointe P, Hutt DM, Koulov AV, Coppinger J, Gurkan C, Kellner W, Matteson J, Plutner $\mathrm{H}$, et al. 2006. Hsp90 cochaperone Aha1 downregulation rescues misfolding of CFTR in cystic fibrosis. Cell 127: $803-815$.

Wellhauser L, Kim Chiaw P, Pasyk S, Li C, Ramjeesingh M, Bear CE. 2009. A small-molecule modulator interacts directly with deltaPhe508-CFTR to modify its ATPase activity and conformational stability. Mol Pharmacol 75: $1430-1438$.

Wine JJ. 2010. The development of lung disease in cystic fibrosis pigs. Sci Transl Med 2: 29.

Wiseman RL, Powers ET, Buxbaum JN, Kelly JW, Balch WE. 2007. An adaptable standard for protein export from the endoplasmic reticulum. Cell 131: 809-821.

Wright JM, Nikolsky Y, Serebryiskaya T, Wetmore DR. 2009. MetaMiner (CF): a disease-oriented bioinformatics analysis environment. Methods Mol Biol 563: 353-367.

Youker RT, Walsh P, Beilharz T, Lithgow T, Brodsky JL. 2004. Distinct roles for the Hsp40 and Hsp90 molecular chaperones during cystic fibrosis transmembrane conductance regulator degradation in yeast. Mol Biol Cell 15: 4787-4797.

Younger JM, Chen L, Ren HY, Rosser MF, Turnbull EL, Fan CY, Patterson C, Cyr DM. 2006. Sequential qualitycontrol checkpoints triage misfolded cystic fibrosis transmembrane conductance regulator. Cell 126: 571-582.

Younger JM, Ren HY, Chen L, Fan CY, Fields A, Patterson C, Cyr DM. 2004. A foldable CFTR $\{$ Delta $\}$ F508 biogenic intermediate accumulates upon inhibition of the Hsc70-CHIP E3 ubiquitin ligase. J Cell Biol 167: 10751085.

Yu Z, Sawkar AR, Kelly JW. 2007. Pharmacologic chaperoning as a strategy to treat Gaucher disease. FEBS J 274: 4944-4950.

Zemanick ET, Harris JK, Conway S, Konstan MW, Marshall B, Quittner AL, Retsch-Bogart G, Saiman L, Accurso FJ. 2010. Measuring and improving respiratory outcomes in cystic fibrosis lung disease: opportunities and challenges to therapy. J Cyst Fibros 9: 1-16.

Zhang K, Kaufman RJ. 2008. From endoplasmic-reticulum stress to the inflammatory response. Nature 454: 455462.

Zhang Y, Nijbroek G, Sullivan ML, McCracken AA, Watkins SC, Michaelis S, Brodsky JL. 2001. Hsp70 molecular chaperone facilitates endoplasmic reticulum-associated protein degradation of cystic fibrosis transmembrane conductance regulator in yeast. Mol Biol Cell 12: 1303-1314.

Zhang H, Schmidt BZ, Sun F, Condliffe SB, Butterworth MB, Youker RT, Brodsky JL, Aridor M, Frizzell RA. 2006. Cysteine string protein monitors late steps in cystic fibrosis transmembrane conductance regulator biogenesis. J Biol Chem 281: 11312-11321. 


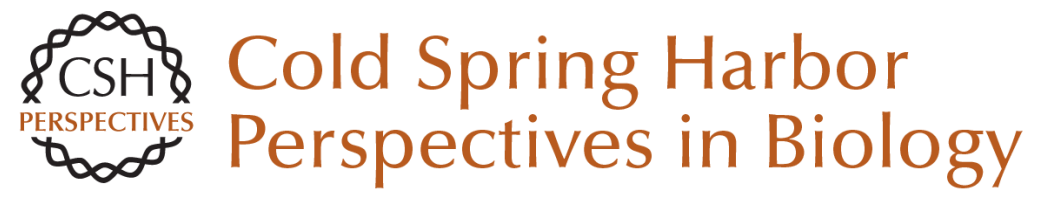

\title{
Emergent Properties of Proteostasis in Managing Cystic Fibrosis
}

\author{
William E. Balch, Daniela M. Roth and Darren M. Hutt
}

Cold Spring Harb Perspect Biol 2011; doi: 10.1101/cshperspect.a004499 originally published online December 15, 2010

\section{Subject Collection Protein Homeostasis}

Proteome-Scale Mapping of Perturbed

Proteostasis in Living Cells

Isabel Lam, Erinc Hallacli and Vikram Khurana

Pharmacologic Approaches for Adapting Proteostasis in the Secretory Pathway to

Ameliorate Protein Conformational Diseases Jeffery W. Kelly

Cell-Nonautonomous Regulation of Proteostasis in Aging and Disease

Richard I. Morimoto

The Autophagy Lysosomal Pathway and

Neurodegeneration

Steven Finkbeiner

Functional Modules of the Proteostasis Network Gopal G. Jayaraj, Mark S. Hipp and F. Ulrich Hartl

Protein Solubility Predictions Using the CamSol Method in the Study of Protein Homeostasis Pietro Sormanni and Michele Vendruscolo

Recognition and Degradation of Mislocalized

Proteins in Health and Disease

Ramanujan S. Hegde and Eszter Zavodszky

The Nuclear and DNA-Associated Molecular Chaperone Network

Zlata Gvozdenov, Janhavi Kolhe and Brian C. Freeman
The Amyloid Phenomenon and Its Significance in Biology and Medicine

Christopher M. Dobson, Tuomas P.J. Knowles and Michele Vendruscolo

A Chemical Biology Approach to the Chaperome

in Cancer--HSP9O and Beyond

Tony Taldone, Tai Wang, Anna Rodina, et al.

Proteostasis in Viral Infection: Unfolding the Complex Virus-Chaperone Interplay Ranen Aviner and Judith Frydman

The Proteasome and Its Network: Engineering for Adaptability Daniel Finley and Miguel A. Prado

Functional Amyloids Daniel Otzen and Roland Riek

Chaperone Interactions at the Ribosome Elke Deuerling, Martin Gamerdinger and Stefan G. Kreft

Mechanisms of Small Heat Shock Proteins Maria K. Janowska, Hannah E.R. Baughman, Christopher N. Woods, et al.

Structure, Function, and Regulation of the Hsp90 Machinery

Maximilian M. Biebl and Johannes Buchner

For additional articles in this collection, see http://cshperspectives.cshlp.org/cgi/collection/

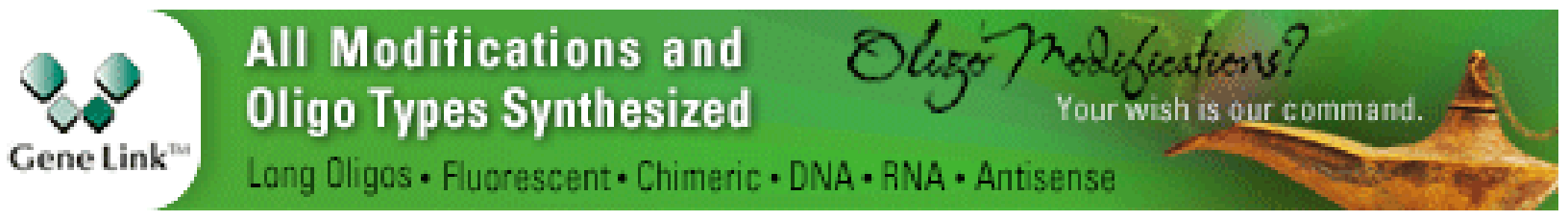


For additional articles in this collection, see http://cshperspectives.cshlp.org/cgi/collection/

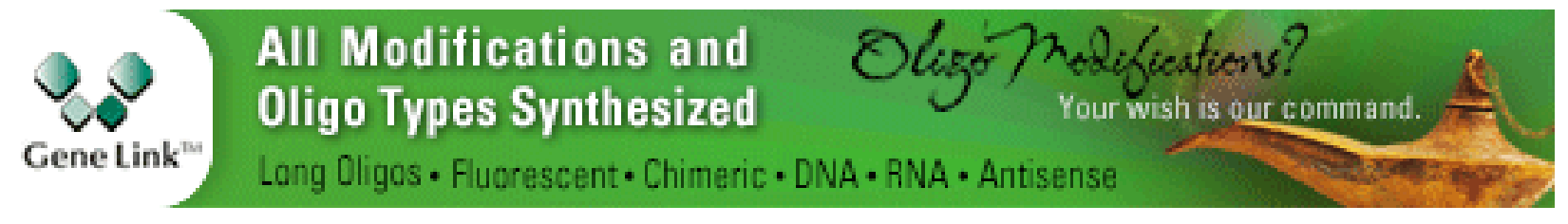

Copyright @ 2011 Cold Spring Harbor Laboratory Press; all rights reserved 